\title{
Physical and electrochemical aspects of bio- and nanomaterials
}

\author{
Libuše Trnková $^{1} \cdot$ Zdeněk Farka $^{2}$
}

Received: 17 February 2016/ Accepted: 20 February 2016/Published online: 9 March 2016

(C) Springer-Verlag Wien 2016

This Special Issue of Chemical Monthly is dedicated to contributions presented at the 15th Workshop of Physical Chemists and Electrochemists (WOPCE) which took place in Brno, Czech Republic, in May 2015. This two-day conference has been organized since 2000, and the last 3 years of the WOPCE were held in the new campus of Masaryk University. All participants presented the latest research in an inspiring lecture session or in the traditionally highly stimulating poster session. The conference scope changes slightly throughout the years, but physical chemistry together with electrochemistry always remains as the main WOPCE topic. The aim of this meeting is to show students and young researchers that physical chemistry is broadly defined and of fundamental importance for the development of interdisciplinary areas that link synthetic, structural, biological, materials and theoretical chemistry together. As the organizers of this meeting, we want to underline the fact that the development of science contributes to intensified interdisciplinary collaboration, thus closely linking scientific research in the fields of both life and materials science. An integral part of the WOPCE is the Young Researchers' Section which gives the opportunity not only to present and discuss results, but also to compete for the best and most interesting presentation.
We would like to devote this Special Issue to Professor Petr Zuman, who has had an immense and longstanding impact on electrochemical science and celebrated his 90th birthday in January 2016. He is a distinguished emeritus research professor of chemistry at Clarkson University. He has still continued actively collaborating and publishing. Prof. Petr Zuman significantly contributed to the description of numerous mechanisms of organic reactions investigated by polarographic, voltammetric, and kinetic measurements.

We thank all the authors who submitted their results to be published in this issue and we do hope that the readers will find this Special Issue of great interest. A common link of the publications are the physical and electrochemical aspects of bio- and nanomaterials. The main focus has been paid on the properties and interactions of DNA. Starting from electrochemical analyses, through expression up to its visualization by AFM, all of them are covered in this issue. The materials science is mainly represented by synthesis and different application of various nanostructure types. The overlap of life and materials science is demonstrated by biological application of different structures.
Libuše Trnková

libuse@chemi.muni.cz

1 Department of Chemistry, Masaryk University, Kamenice 5, 62500 Brno, Czech Republic

2 CEITEC MU, Masaryk University, Kamenice 5, 62500 Brno, Czech Republic 